\title{
Nonlinear soliton matching between optical fibers
}

\author{
Agger, Christian; Sørensen, Simon Toft; Thomsen, Carsten L.; Keiding, Søren R.; Bang, Ole
}

Published in:

Optics Letters

Link to article, DOI:

10.1364/OL.36.002596

Publication date:

2011

Document Version

Publisher's PDF, also known as Version of record

Link back to DTU Orbit

Citation (APA):

Agger, C., Sørensen, S. T., Thomsen, C. L., Keiding, S. R., \& Bang, O. (2011). Nonlinear soliton matching between optical fibers. Optics Letters, 36(13), 2596-2598. https://doi.org/10.1364/OL.36.002596

\section{General rights}

Copyright and moral rights for the publications made accessible in the public portal are retained by the authors and/or other copyright owners and it is a condition of accessing publications that users recognise and abide by the legal requirements associated with these rights.

- Users may download and print one copy of any publication from the public portal for the purpose of private study or research.

- You may not further distribute the material or use it for any profit-making activity or commercial gain

- You may freely distribute the URL identifying the publication in the public portal

If you believe that this document breaches copyright please contact us providing details, and we will remove access to the work immediately and investigate your claim. 


\title{
Nonlinear soliton matching between optical fibers
}

\author{
Christian Agger, ${ }^{1, *}$ Simon T. Sørensen, ${ }^{1}$ Carsten L. Thomsen, ${ }^{2}$ Søren R. Keiding, ${ }^{3}$ and Ole Bang ${ }^{1,2}$ \\ ${ }^{1}$ DTU Fotonik, Department of Photonics Engineering, Technical University of Denmark, \\ 2800 Kongens Lyngby, Denmark \\ ${ }^{2}$ NKT Photonics A/S, Blokken 84, 3460 Birkerød, Denmark \\ ${ }^{3}$ Department of Chemistry, Aarhus University, 8000 Aarhus C, Denmark \\ ${ }^{*}$ Corresponding author: cagg@fotonik.dtu.dk.
}

Received May 2, 2011; accepted May 28, 2011;

posted June 8, 2011 (Doc. ID 146776); published July 1, 2011

\begin{abstract}
In this Letter, we propose a generic nonlinear coupling coefficient, $\eta_{\mathrm{NL}}^{2}=\eta\left|\gamma / \beta_{2}\right|_{\text {fiber } 2} /\left|\gamma / \beta_{2}\right|_{\text {fiber1 }}$, which gives a quantitative measure for the efficiency of nonlinear matching of optical fibers by describing how a fundamental soliton couples from one fiber into another. Specifically, we use $\eta_{\mathrm{NL}}$ to demonstrate a significant soliton selffrequency shift of a fundamental soliton, and we show that nonlinear matching can take precedence over linear mode matching. The nonlinear coupling coefficient depends on both the dispersion $\left(\beta_{2}\right)$ and nonlinearity $(\gamma)$, as well as on the power coupling efficiency $\eta$. Being generic, $\eta_{\mathrm{NL}}$ enables engineering of general waveguide systems, e.g., for optimized Raman redshift or supercontinuum generation. (c) 2011 Optical Society of America

OCIS codes: $190.4370,190.5650,060.4370,060.5530$.
\end{abstract}

When coupling linear waves between optical waveguides, mode-field diameter (MFD) matching is important to avoid loss of power. When coupling fundamental solitons between two nonlinear fibers, however, soliton number preservation becomes an equally important matching requirement, placing restrictions not only on the dispersion of the fiber $\left(\beta_{2}\right)$, but also on their nonlinearity $(\gamma)$. Nonlinear soliton matching (NLSM) of fibers is becoming increasingly important for optimization of supercontinuum generation (SCG) by concatenating silica fibers [1], and for extending Raman redshift and SCG [2] into the infrared (IR) by concatenating silica and soft-glass fibers.

In this Letter, we show that a matching requirement on the ratio of $\gamma /\left|\beta_{2}\right|$ for the fibers ensures a good coupling of a fundamental soliton from one fiber into another. The proposed scheme is generic, but we use it here to obtain continued redshift by the soliton self-frequency shift (SSFS). In particular, we use the NLSM to study the coupling between silica and soft-glass photonic crystal fibers (PCFs) and find parameter regimes in which a soliton, which is no longer redshifting in a silica fiber due to strong dispersion [1], can couple into a soft-glass fiber for further redshifting into the IR.

Our generic concept of NLSM between two nonlinear fibers is illustrated in Fig. 1. First a fundamental soliton (soliton number $N=N_{1}=1$ ) at pump wavelength $\lambda_{p}$ is launched into fiber 1 where it undergoes an initial redshift (Fig. 1, bottom) until a desired coupling wavelength $\lambda_{c}$. Subsequently the redshifted soliton is coupled into fiber 2, where it has a corresponding soliton number $N=N_{2}$. There are then three scenarios depending on the soliton number $N_{2}$ : (1) If $N_{2}<1 / 2$, there is not enough power to excite a soliton in fiber 2 (Fig. 1, top left). In this case the spectral content of the pulse will not change, but the pulse will spread temporally. (2) If $1 / 2<N_{2}<3 / 2$ (Fig. 1, top middle), the pulse will remain a fundamental soliton in fiber 2 . This case of continued fundamental soliton propagation gives a large conversion of energy from the input pulse to the redshifted soliton. (3) If $N_{2}>3 / 2$
(Fig. 1, top right), a higher-order soliton is generated, which will undergo soliton fission and possibly dispersive wave generation. The breakup reduces the amount of energy converted from the input soliton wavelength to the wavelength of the most redshifted soliton, but it increases the total soliton redshift.

As an example illustrating the NLSM, we consider coupling between silica and soft-glass PCFs with hole diameter $d$, pitch $\Lambda$, and seven rings of air holes [3]. All fiber properties are calculated using a commercially available finite-element tool and material dispersion taken from the literature [4-ㅜ] . Fiber 1 is made from fused silica and we investigate two different materials for fiber 2. Fiber 2a is a PCF made from highly nonlinear tellurite and fiber $2 \mathrm{~b}$ is a fluoride based ZBLAN PCF. The group velocity dispersion (GVD) parameter $\beta_{2}$ and the effective area $A_{\text {eff }}$ of the fibers are shown in Fig. 2 . Fiber

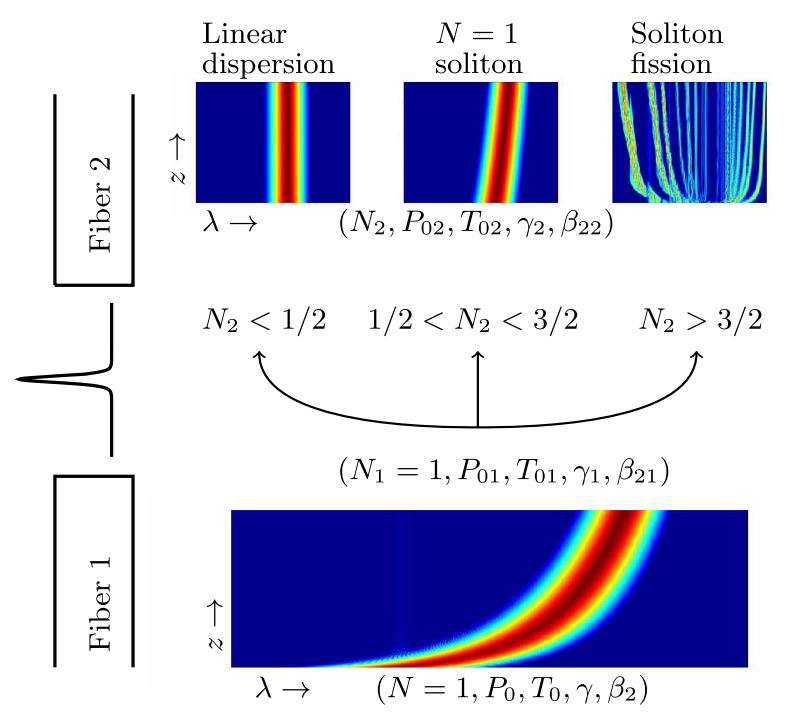

Fig. 1. (Color online) Energy spectral density evolution in a generic fiber system. Initially an $N=1$ soliton redshifts in fiber 1 , and the output is coupled into fiber 2 . 

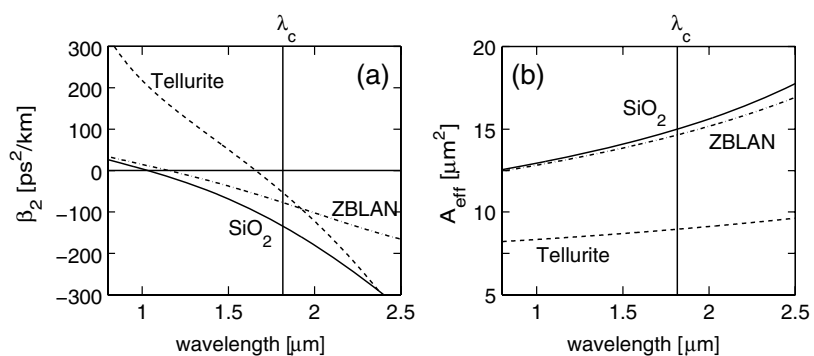

Fig. 2. (a) GVD parameter $\beta_{2}$ and (b) effective area $A_{\text {eff }}$ of the fibers. Vertical line marks $\lambda_{c}=1816 \mathrm{~nm}$. For fiber parameters, see Table $\underline{1}$.

properties, such as zero dispersion wavelength $\lambda_{\mathrm{ZD}}$, are summarized in Table 1 and Table 2.

It is well known that the analytical soliton solutions to the nonlinear Schrödinger equation (NLSE) are characterized by the soliton number $N^{2}=\gamma P_{0} T_{0}^{2} /\left|\beta_{2}\right|$, where $T_{0}=T_{\mathrm{FWHM}} / 1.763$ is the pulse length and $P_{0}$ is the peak power. The nonlinear coefficient is $\gamma=n_{2} \omega /\left[c A_{\text {eff }}\right]$, where $n_{2}$ is the material nonlinear refractive index and $c$ is the speed of light in vacuum. In fibers the governing equation is the generalized NLSE (GNLSE), which includes the Raman effect and the complete dispersion profile. To determine the development of the electric field envelope, the GNLSE is solved numerically using a fourth-order Runge-Kutta integration scheme, where accumulated error is controlled using the local error method [ $[7,8]$. The GNLSE is solved to obtain the contour plots shown both in Figs. 1 and 4 . Because the central wavelength is below $2 \mu \mathrm{m}$ in fiber 1 at all times and because we focus specifically on the effect of the fiber coupling, propagation loss is ignored in both fiber 1 and 2. As an initial condition we use a fundamental soliton with a central wavelength $\lambda_{p}=1550 \mathrm{~nm}$, and FWHM pulse duration of $T_{\mathrm{FWHM}}=50 \mathrm{fs}$, corresponding to an Erbium laser source. In simulations we use the latest reported Raman response functions for each material $[\underline{9}, 10]$. After propagation in $10 \mathrm{~m}$ of fiber 1 , simulation of the GNLSE shows that the soliton has redshifted to $\lambda_{c}=1816 \mathrm{~nm}$ due to the Raman effect [11], while continuously adjusting its pulse width and peak power to preserve $N_{1}=1$ (see Table 2). Using that $N_{1}=1$ we have the relation

Table 1. Fiber Parameters Used in the Setup

\begin{tabular}{llcccc}
\hline Fiber & Material & $\lambda_{\text {ZD }}[\mathrm{nm}]$ & $\Lambda[\mu \mathrm{m}]$ & $d / \Lambda$ & $n_{2}\left[10^{-20} \frac{\mathrm{m}^{2}}{\mathrm{~W}}\right]$ \\
\hline 1 & $\mathrm{SiO}_{2}{ }^{a}$ & 1033 & 3.3 & 0.52 & 2.6 \\
$2 \mathrm{a}$ & $\mathrm{Tellurite}^{a}$ & 1659 & 3.5 & 0.8 & 59 \\
$2 \mathrm{~b}$ & $\mathrm{ZBLAN}^{a}$ & 1154 & 3.3 & 0.52 & 2.2 \\
\hline
\end{tabular}

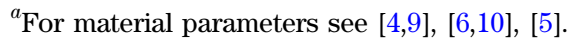

Table 2. Pulse and Derived Parameters Used in Calculations

\begin{tabular}{lcccccc}
\hline Fiber & $j$ & $A_{\mathrm{eff}, j}\left[\mu \mathrm{m}^{2}\right]$ & $\beta_{2 j}\left[\frac{\mathrm{ps}^{2}}{\mathrm{~m}}\right]$ & $T_{\mathrm{FWHM}, j}[\mathrm{fs}]$ & $P_{0 j}[\mathrm{~kW}]$ & $N_{j}$ \\
\hline 1-in & & 14.2 & -77.9 & 50.0 & 13.08 & 1.00 \\
1-out & 1 & 15.0 & -134.2 & 122.5 & 4.58 & 0.99 \\
2a-in & 2 & 9.0 & -53.2 & 122.5 & 4.58 & 9.73 \\
2b-in & 2 & 14.7 & -77.2 & 122.5 & 4.58 & 1.22 \\
\hline
\end{tabular}

$$
N_{1}^{2}=T_{01}^{2} P_{01} \gamma_{1} /\left|\beta_{21}\right|=1,
$$

where $T_{01}\left(P_{01}\right)$ is the pulse width (peak power) of the soliton at the output of fiber 1 (see Fig. 1 for notation). We assume an adiabatic soliton coupling, which preserves the pulse shape when passing the interface, but can decrease the peak power. Thus, it is reasonable to assume that $T_{02}=T_{01}$, where $T_{02}$ is the soliton pulse width at the input of fiber 2 . Fresnel reflection and MFD mismatch dictates that $P_{02}=\eta P_{01}$, where $0 \leq \eta \leq 1$ is the power coupling efficiency. Using this, we define the nonlinear coupling coefficient

$$
\eta_{\mathrm{NL}}^{2} \equiv N_{2}^{2} / N_{1}^{2}=\eta\left(\gamma_{2} /\left|\beta_{22}\right|\right) /\left(\gamma_{1} /\left|\beta_{21}\right|\right),
$$

which is given in terms of material parameters, fiber dispersion, and the power coupling efficiency $\eta$. In the case considered here, when coupling a fundamental soliton from fiber 1 into fiber $2, \eta_{\mathrm{NL}}$ is equivalent to the factor by which the soliton number changes across the interface. For further propagation of a stable soliton in fiber 2 , it is required that $1 / 2<\eta_{\mathrm{NL}}<3 / 2$. In Fig. 3 we show $\eta_{\mathrm{NL}}$ for fibers $2 \mathrm{a}$ and $2 \mathrm{~b}$ as function of wavelength. Notice that $\eta_{\mathrm{NL}}$ is singular at $\beta_{22}=0$, thus $\eta_{\mathrm{NL}} \rightarrow \infty$ at $\lambda_{\mathrm{ZD}}$ of fiber 2 . The black horizontal solid lines mark the band of NLSM, where continued SSFS of a stable fundamental soliton can be expected. The power coupling efficiency due to imperfect matching of the MFD of Gaussian shaped modes can be estimated from [12]

$$
\eta_{\mathrm{MFD}}=4\left(A_{\mathrm{eff}, 1} A_{\mathrm{eff}, 2}\right)^{2} /\left(A_{\mathrm{eff}, 1}^{2}+A_{\mathrm{eff}, 2}^{2}\right)^{2} .
$$

It should be noted that the modes in PCFs are not Gaussian shaped, but we use Eq. (3) as an approximation to the coupling efficiency. Using the effective areas from Table 2 gives a power coupling efficiency of $\eta_{\mathrm{MFD}}=77 \%$ for the $\mathrm{SiO}_{2}$-tellurite coupling and $\eta_{\mathrm{MFD}}=99 \% \approx 1$ for the $\mathrm{SiO}_{2}$-ZBLAN coupling, both at $\lambda=\lambda_{c}=1816 \mathrm{~nm}$. As indicated by Eq. (2), inclusion of the power coupling efficiency lowers the NLSM curve by a factor of $\sqrt{\eta_{\mathrm{MFD}}}$. Figure 3 shows the NLSM curve for the $\mathrm{SiO}_{2}$-tellurite coupling both with $\eta=1$ and $\eta=\eta_{\mathrm{MFD}}$ calculated from Eq. (3). By design of the waveguide dispersion and choice of nonlinear fiber material, it is possible to

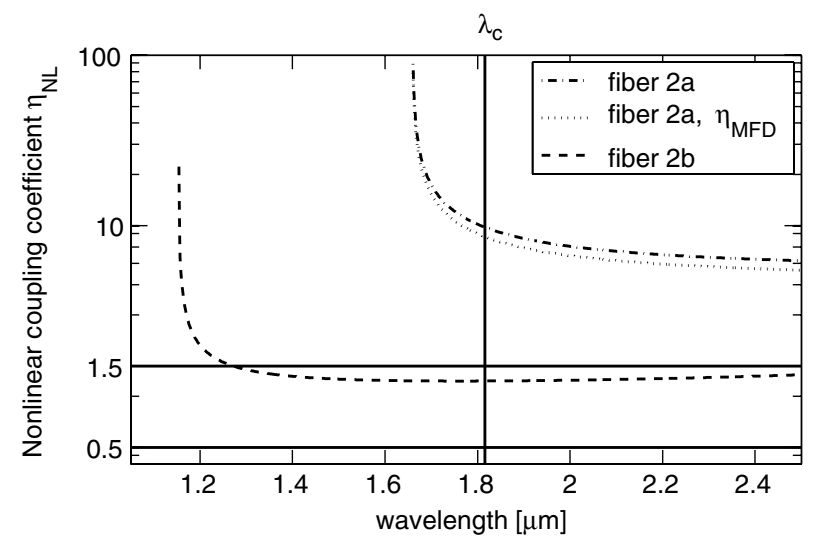

Fig. 3. Nonlinear coupling coefficient, $\eta_{\mathrm{NL}}$, for fiber $2 \mathrm{a}$ (dash dotted) and fiber $2 \mathrm{~b}$ (dashed) with $\eta=1$. For fiber $2 \mathrm{a}$ is also shown $\eta_{\mathrm{NL}}$, with $\eta=\eta_{\mathrm{MFD}}\left(\lambda_{c}\right)$. The black vertical line marks $\lambda_{c}=1816 \mathrm{~nm}$. 

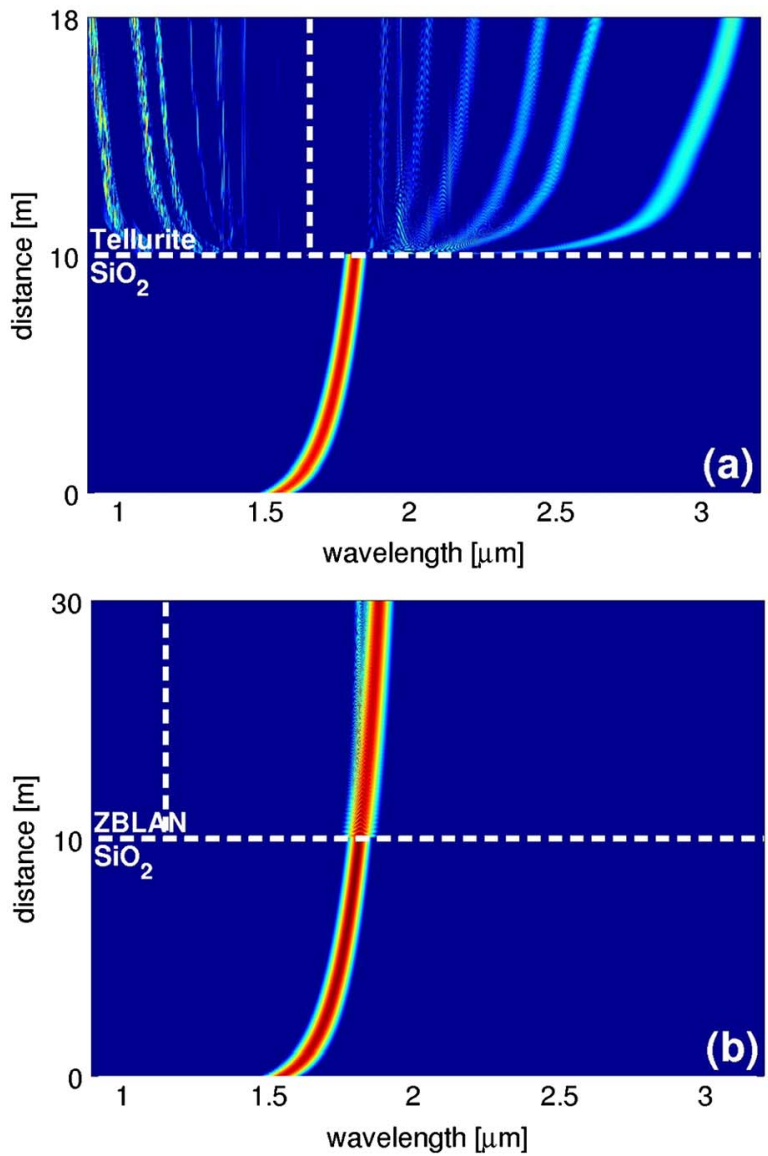

Fig. 4. (Color online) Evolution of the energy spectral density in (a) fiber 1 and fiber $2 \mathrm{a}$ and (b) fiber 1 and fiber $2 \mathrm{~b}$. In (a) $\eta_{\mathrm{NL}}=9.73 \gg 3 / 2$, and a higher-order soliton undergoes soliton fission in fiber $2 \mathrm{a}$. In (b) $\eta_{\mathrm{NL}}=1.22$, and a fundamental soliton propagates throughout the system. In both simulations the power coupling efficiency is $\eta=1$. White vertical line marks $\lambda_{\text {ZD }}$ of fiber 2 .

compensate for the linear power reduction from MFD mismatch. We thus conclude that NLSM takes precedence over mode matching. In an experimental setting, additional loss mechanisms can be accounted for by calculating an overall power coupling efficiency $\eta$ at the interface, taking into account, e.g., Fresnel loss.

In Fig. $\underline{3}$, we find that $\eta_{\mathrm{NL}} \gg 3 / 2$ both for $\eta=1$ and $\eta=$ $\eta_{\text {MFD }}$ when coupling into fiber $2 \mathrm{a}$. Consequently, we expect soliton fission will occur in fiber $2 \mathrm{a}$ after coupling from fiber 1. Simulation results for propagation in $10 \mathrm{~m}$ of fiber 1 and $8 \mathrm{~m}$ in fiber 2a are shown in Fig. 4(a). Here it is clearly confirmed that after a short distance of propagation in fiber $2 \mathrm{a}$, soliton fission causes a breakup of the pulse, and generates multiple separated solitons. The central wavelength of the most redshifted soliton becomes $\lambda=3103 \mathrm{~nm}$ in this case, and approximately $18 \%$ of the energy in the input soliton has been converted to this wavelength. If, on the other hand, the output from fiber 1 is coupled into fiber $2 b$, where the NLSM condition is satisfied so that $1 / 2<\eta_{\mathrm{NL}}<3 / 2$, a fundamental soliton will continue propagation and redshift. This is also confirmed by simulations in Fig. 4(b). Here it is shown that the fundamental soliton from fiber 1 continues stable redshift in fiber $2 \mathrm{~b}$, and the central wavelength increases from $\lambda_{c}=1816 \mathrm{~nm}$ to $1857 \mathrm{~nm}$. In this case approximately $83 \%$ of the energy from the input pulse is converted to this wavelength.

In conclusion, we have proposed a measure, $\eta_{\mathrm{NL}}$, describing the coupling of fundamental solitons across a generic fiber interface. Because the proposed NLSM scheme depends both on material and fiber parameters, it is an important design tool for optimization of fiber waveguide systems for, e.g., large redshifts of solitons. The usefulness of our scheme is demonstrated in a fiber system that enables a redshift of $300 \mathrm{~nm}$ for a $50 \mathrm{fs}$ fundamental soliton at $\lambda_{p}=1550 \mathrm{~nm}$ with peak power $P_{0}=13.08 \mathrm{~kW}$. We find an energy conversion efficiency above $80 \%$ in $30 \mathrm{~m}$ of concatenated fibers. Also demonstrated is full octave spanning redshift of the same initial soliton, where around $20 \%$ of the energy is converted to $3100 \mathrm{~nm}$ in $18 \mathrm{~m}$ of fiber. We have shown that in this scheme matching dispersion and nonlinearity combined takes precedence over MFD matching. We speculate that designing fiber systems with a broad wavelength range satisfying the NLSM condition is useful for extending supercontinuum into the IR. This will be subject to more research.

The authors acknowledge support from the Danish National Advanced Technology Foundation.

\section{References}

1. J. C. Travers, S. V. Popov, and J. R. Taylor, Opt. Lett. 30, 3132 (2005).

2. C. Xia, Z. Xu, M. Islam, F. Terry, M. Freeman, A. Zakel, and J. Mauricio, IEEE J. Sel. Top. Quantum Electron. 15, 422 (2009).

3. P. S. Russell, J. Lightwave Technol. 24, 4729 (2006).

4. K. Okamoto, Fundamentals of Optical Waveguides (Elsevier, 2006).

5. F. Gan, J. Non-Cryst. Solids 184, 9 (1995).

6. M. D. O'Donnell, K. Richardson, R. Stolen, A. B. Seddon, D. Furniss, V. K. Tikhomirov, C. Rivero, M. Ramme, R. Stegeman, G. Stegeman, M. Couzi, and T. Cardinal, J. Am. Ceram. Soc. 90, 1448 (2007).

7. O. V. Sinkin, R. Holzlöhner, J. Zweck, and C. R. Menyuk, J. Lightwave Technol. 21, 61 (2003).

8. M. H. Frosz, Opt. Express 18, 14778 (2010).

9. G. Agrawal, Nonlinear Fiber Optics, 4th ed. (Academic, 2006).

10. D. Buccoliero, H. Steffensen, O. Bang, H. EbendorffHeidepriem, and T. M. Monro, Appl. Phys. Lett. 97, 061106 (2010).

11. F. M. Mitschke and L. F. Mollenauer, Opt. Lett. 11, 659 (1986).

12. D. Marcuse, Bell Syst. Tech. J. 56, 703 (1977). 